Bull. Austral. Math. Soc.

$47 \mathrm{~A} 11,47 \mathrm{~B} 20,47 \mathrm{~B} 47$

VOL. 66 (2002) [425-441]

\title{
ON TOTALLY PARANORMAL OPERATORS
}

\section{Christoph SCHMOEger}

A continuous linear operator on a complex Banach space is said to be paranormal if $\|T x\|^{2} \leqslant\left\|T^{2} x\right\|\|x\|$ for all $x \in X . T$ is called totally paranormal if $T-\lambda$ is paranormal for every $\lambda \in \mathcal{C}$. In this paper we investigate the class of totally paranormal operators. We shall see that Weyl's theorem holds for operators in this class. We also show that for totally paranormal operators the Weyl spectrum satisfies the spectral mapping theorem. In Section 5 of this paper we investigate the operator equations $e^{T}=e^{S}$ and $e^{T} e^{S}=e^{S} e^{T}$ for totally paranormal operators $T$ and $S$.

\section{General and introductory material}

Throughout this paper let $X$ be a complex Banach space and denote the set of bounded linear operators on $X$ by $\mathcal{L}(X)$. Let $\sigma(T)$ and $\rho(T)$ denote, respectively, the spectrum and the resolvent set of an element $T$ of $\mathcal{L}(X)$. By $r(T)$ we denote the spectral radius of $T$ and by $\sigma_{p}(T)$ the set of eigenvalues of $T$. The set of those operators $T$ in $\mathcal{L}(X)$ for which the range $T(X)$ is closed and $\alpha(T)$, the dimension of the kernel $N(T)$ of $T$, is finite is denoted by $\Phi_{+}(X)$. Set

$$
\Phi_{-}(X)=\{T \in \mathcal{L}(X): \beta(T) \text { is finite }\},
$$

where $\beta(T)$ is the codimension of $T(X)$. Observe that $T(X)$ is closed if $T \in \Phi_{-}(X)([9$, Satz 55.4]). Operators in $\Phi_{+}(X) \cup \Phi_{-}(X)$ are called semi-Fredholm-operators. For such an operator $T$ we define the index of $T$ by

$$
\operatorname{ind}(T)=\alpha(T)-\beta(T) .
$$

An operator $T$ is called a Fredholm operator if $T \in \Phi(X)=\Phi_{+}(X) \cap \Phi_{-}(X)$.

Let $T \in \mathcal{L}(X)$. Define $p(T)$ [respecitvely $q(T)$ ], the ascent [respectively descent] of $T$, to be the smallest integer $n \geqslant 0$ such that

$$
N\left(T^{n+1}\right)=N\left(T^{n}\right) \quad\left[\text { respectively } T^{n+1}(X)=T^{n}(X)\right]
$$

or $\infty$ if no such $n$ exists.

The assertions of the following proposition are shown in $[9$, Section 72 and Section 101].

Proposition 1.1. Let $T \in \mathcal{L}(X)$.

Received 21st March, 2002

Copyright Clearance Centre, Inc. Serial-fee code: 0004-9727/02 \$A2.00+0.00. 
(1) If $p(T)<\infty$ and $q(T)<\infty$, then $p(T)=q(T)$ and $\alpha(T)=\beta(T)$.

(2) If $\alpha(T)=\beta(T)<\infty$ and if $p(T)<\infty$ or $q(T)<\infty$, then $p(T)=q(T)$.

(3) If $p(T)<\infty$, then $\alpha(T) \leqslant \beta(T)$.

(4) If $q(T)<\infty$, then $\beta(T) \leqslant \alpha(T)$.

(5) $\lambda_{0} \in \mathcal{C}$ is a pole of the resolvent $(\lambda-T)^{-1}$ if and only if $p\left(\lambda_{0}-T\right)$ $=q\left(\lambda_{0}-T\right)<\infty$. In this case $p\left(\lambda_{0}-T\right)$ is the order of the pole and $\lambda_{0} \in \sigma_{p}(T)$.

Now we introduce the class of operators which we shall investigate in this paper:

An operator $T \in \mathcal{L}(X)$ is said to be paranormal if

$$
\|T x\|^{2} \leqslant\left\|T^{2} x\right\|\|x\| \text { for all } x \in X .
$$

By $\mathcal{P N}(X)$ we denote the set of all paranormal operators in $\mathcal{L}(X)$. The class $\mathcal{T} \mathcal{P} \mathcal{N}(X)$ of all totally paranormal operators is given by

$$
\mathcal{T P N}(X)=\{T \in \mathcal{L}(X): \lambda-T \in \mathcal{P N}(X) \text { for all } \lambda \in \mathcal{C}\}
$$

Examples 1.2. Let $H$ denote a complex Hilbert space.

(1) $T \in \mathcal{L}(H)$ is said to be hyponormal if

$$
\left\|T^{*} x\right\| \leqslant\|T x\| \quad \text { for every } x \in H \text {. }
$$

If $T$ is hyponormal, then

$$
\|T x\|^{2}=(T x \mid T x)=\left(T^{*} T x \mid x\right) \leqslant\left\|T^{*} T x\right\|\|x\| \leqslant\left\|T^{2} x\right\|\|x\|
$$

for any $x \in H$ (where $(\cdot \mid \cdot)$ denotes the inner product on $H$ ). Thus $T$ is paranormal. It is easy to see that if $T$ is hyponormal, then $T-\lambda$ is hyponormal for each $\lambda \in \mathcal{C}$. Hence every hyponormal operator is totally paranormal.

(2) An operator $T \in \mathcal{L}(H)$ is called subnormal if $T$ has a normal extension. We see from [3, Proposition III 4.2] or [8, p. 108] that subnormal operators are hyponormal. Hence subnormal operators belong to $\mathcal{T} \mathcal{P N}(H)$.

(3) $T \in \mathcal{L}(H)$ is said to be quasinormal if $T$ and $T^{*} T$ commute. Quasinormal operators are subnormal ([3, Proposition III 1.7]), thus they are totally paranormal.

REMARK. In [5] an example of a paranormal operator is constructed which is not totally paranormal.

Proposition 1.3. Let $T \in \mathcal{P N}(X)$.

(1) $T^{n} \in \mathcal{P N}(X)$ for all $n \in \mathcal{N}$.

(2) $\|T\|=r(T)$.

(3) $p(T) \leqslant 1$. 
PROOF:

(1) is shown in [9, Hilfssatz 102.1].

(2) For a unit vector $x \in X,\|T x\|^{2} \leqslant\left\|T^{2} x\right\| \leqslant\left\|T^{2}\right\|$, thus $\|T\|^{2} \leqslant\left\|T^{2}\right\|$ $\leqslant\|T\|^{2}$. By (1) and induction we see that

$$
\left\|T^{2^{n}}\right\|=\|T\|^{2^{n}} \text { for all } n \in \mathcal{N},
$$

hence

$$
r(T)=\lim _{n \rightarrow \infty}\left\|T^{2^{n}}\right\|^{1 / 2^{n}}=\|T\| .
$$

(3) The inclusion $N(T) \subseteq N\left(T^{2}\right)$ is trivial. The inclusion $N\left(T^{2}\right) \subseteq N(T)$ follows from the definition of $\mathcal{P N}(X)$.

COROLlary 1.4. If $T \in \mathcal{T P N}(X)$ then

$$
p(\lambda-T) \leqslant 1 \text { for every } \lambda \in \mathcal{C} .
$$

The present paper is organised as follows: in the next section we are concerned with several essential spectra of totally paranormal operators (Fredholm spectrum, Weyl spectrum, Kato spectrum). In Section 3 we briefly review some concepts of local spectral theory and investigate local spectral properties of operators in $\mathcal{T P \mathcal { N }}(X)$. In Section 4 we collect some results concerning the inner derivation determined by $T \in \mathcal{L}(X)$. These results will be used in Section 5, where we consider the exponential function

$$
e^{T}=\sum_{n=0}^{\infty} \frac{T^{n}}{n !}
$$

with $T \in \mathcal{T P N}(X)$. In the final section of this paper we shall see that our results of Section 5 are still valid for scalar-type operators (in the sense of Dunford).

\section{ESSENTIAL SPECTRA OF TOTALLY PARANORMAL OPERATORS}

For an operator $T \in \mathcal{L}(X)$ we shall use the following notations:

$$
\begin{aligned}
\Phi(T) & =\{\lambda \in \mathcal{C}: \lambda-T \in \Phi(X)\}, \\
\Sigma(T) & =\{\lambda \in \mathcal{C}: \lambda-T \text { is semi-Fredholm }\}, \\
\sigma_{F}(T) & =\mathcal{C} \backslash \Phi(T)
\end{aligned}
$$

and

$$
\sigma_{s F}(T)=\mathcal{C} \backslash \Sigma(T) .
$$

A Fredholm operator $T$ with ind $(T)=0$ is called a Weyl operator. The Weyl spectrum of $T \in \mathcal{L}(X)$ is defined to be

$$
\sigma_{W}(T)=\{\lambda \in \mathcal{C}: \lambda-T \text { is not a Weyl operator }\} .
$$


It is well known that $\Phi(T)$ and $\Sigma(T)$ are open ([9, Section 82]) and that $\sigma_{W}(T)$ is non empty and compact (see $[\mathbf{1}, \mathbf{2}])$.

For $T \in \mathcal{L}(X)$ we denote by $\pi_{00}(T)$ the set of isolated points of $\sigma(T)$ which are eigenvalues of finite multiplicity.

Following Coburn [2], we say that Weyl's theorem holds for $T \in \mathcal{L}(X)$ if

$$
\sigma_{W}(T)=\sigma(T) \backslash \pi_{00}(T) .
$$

There are several classes of operators, including hyponormal operators on a Hilbert space, for which Weyl's theorem holds (see $[1,2]$ ).

Proposition 2.1. Let $T \in \mathcal{P N}(X)$. Then

$$
T \in \Phi(X) \text { if and only if } T \in \Phi_{-}(X) \text {. }
$$

In this case ind $(T) \leqslant 0$.

Proof: We only have to show that $T \in \Phi_{-}(X)$ implies $T \in \Phi(X)$ and ind $(T) \leqslant 0$. Since $T \in \mathcal{P N}(X), p(T) \leqslant 1$, by Proposition $1.3(3)$. Thus $\alpha(T) \leqslant \beta(T)$, by Proposition $1.1(3)$. Hence $T \in \Phi(X)$ and $\operatorname{ind}(T) \leqslant 0$.

Corollary 2.2. Suppose that $T \in \mathcal{T P N}(X)$.

(1) $\sigma_{F}(T)=\{\lambda \in \mathcal{C}: \beta(\lambda-T)=\infty\}$.

(2) If $C$ is a connected component of $\Phi(T)$ then there are exactly the following two possibilities:

(i) $\operatorname{ind}(\lambda-T)=0$ and $p(\lambda-T)=q(\lambda-T) \leqslant 1$ for all $\lambda \in C$,

(ii) $\quad$ ind $(\lambda-T)<0, p(\lambda-T) \leqslant 1$ and $q(\lambda-T)=\infty$ for all $\lambda \in C$.

Proof: (1) follows from Proposition 2.1.

(2) Use Corollary 1.4 and Satz 104.6 in [9].

Corollary 2.3. If $T \in \mathcal{T P \mathcal { N }}(X)$ then

$$
\rho(T)=\{\lambda \in \mathcal{C}: \lambda-T \text { is surjective }\} .
$$

Proof: If $\lambda-T$ is surjective, $q(\lambda-T)=0=\beta(\lambda-T)$. Corollary 2.2 shows that $\lambda \in \Phi(T)$ and $p(\lambda-T)=0$. Hence $\lambda-T$ is injective.

Proposition 2.4. Suppose that $T \in \mathcal{L}(X)$ is paranormal and that 0 is an isolated point of $\sigma(T)$. Then $0 \in \sigma_{p}(T)$ and 0 is a pole of order 1 of the resolvent $(\lambda-T)^{-1}$.

Proof: There is some $r>0$ such that the punctured disc

$$
\dot{U}_{r}(0)=\{\lambda \in \mathcal{C}: 0<|\lambda|<r\} \subseteq \rho(T) .
$$

Put $\gamma(t):=r e^{i t}, t \in[0,2 \pi]$, and define the operator $P \in \mathcal{L}(X)$ by

$$
P=\frac{1}{2 \pi i} \int_{\gamma}(\lambda-T)^{-1} d \lambda .
$$


It follows from [9, Satz 100.1] that $P^{2}=P, T P=P T$, so $T(P(X)) \subseteq P(X)$, and $\sigma\left(\left.T\right|_{P(X)}\right)=\{0\}$. Since $T$ is paranormal, $\left.T\right|_{P(X)}$ is paranormal on the Banach space $P(X)$, thus, by Proposition 1.3(2),

$$
\left\|\left.T\right|_{P(X)}\right\|=r\left(\left.T\right|_{P(X)}\right)=0 .
$$

This gives $T P=0$.

As an isolated point of $\sigma(T), 0$ is a non-removable singularity of $(\lambda-T)^{-1}$, hence $(\lambda-T)^{-1}$ has the Laurent expansion

$$
(\lambda-T)^{-1}=\sum_{n=1}^{\infty} \frac{P_{n}}{\lambda^{n}}+\sum_{n=0}^{\infty} \lambda^{n} Q_{n}
$$

in $\dot{U}_{r}(0)$ with $P_{n}, Q_{n} \in \mathcal{L}(X)$. It is seen from [9, (101.9)] that

$$
P_{1}=P \quad \text { and } \quad P_{n}=T^{n-1} P \quad(n=1,2, \ldots) .
$$

Since $T P=0, P_{n}=0$ for $n \geqslant 2$. This shows that 0 is a pole of order 1 of $(\lambda-T)^{-1}$. From Proposition 1.1(5) and Proposition 1.3(3) we get $p(T)=q(T)=1$ and $0 \in \sigma_{p}(T)$.

Theorem 2.5. If $T \in \mathcal{T} \mathcal{P N}(X)$, then Weyl's theorem holds for $T$.

Proof: First we show that $\sigma_{W}(T) \subseteq \sigma(T) \backslash \pi_{00}(T)$. Take $\lambda \in \sigma_{W}(T)$. Since $T-\lambda$ is paranormal, we can assume that $\lambda=0$. Suppose to the contrary that $0 \in \pi_{00}(T)$. Then 0 is an isolated point of $\sigma(T)$ and $\alpha(T)<\infty$. Proposition 2.4 and Proposition 1.1 show that $p(T)=q(T)=1$ and $\beta(T)=\alpha(T)<\infty$. Hence $T$ is Fredholm and $\operatorname{ind}(T)=0$. This contradicts $0 \in \sigma_{W}(T)$.

Now we show that $\sigma(T) \backslash \pi_{00}(T) \subseteq \sigma_{W}(T)$. Take $\lambda \in \sigma(T) \backslash \pi_{00}(T)$ and suppose that $\lambda \notin \sigma_{W}(T)$. As above we assume $\lambda=0$. Hence $T$ is a Weyl operator. Proposition 2.2(2) yields $p(T)=q(T)=1$, thus 0 is an isolated point of $\sigma(T)$ and $0 \in \sigma_{p}(T)$ (Proposition 1.1(5)). Since $T \in \Phi(X), \alpha(T)<\infty$, therefore $0 \in \pi_{00}(T)$, a contradiction.

An operator $T \in \mathcal{L}(X)$ is called isoloid if isolated points of $\sigma(T)$ are eigenvalues of $T$.

Corollary 2.6. If $T \in \mathcal{T P N}(X)$, then $T$ is isoloid.

Proof: Proposition 2.4.

Before we state the main results of this section we need the following notation for $T \in \mathcal{L}(X):$

$$
\mathcal{H}(T)=\{f: \Delta(f) \rightarrow \mathcal{C}: \Delta(f) \text { is open, } \sigma(T) \subseteq \Delta(f), \quad f \text { is holomorphic }\} .
$$

For $f \in \mathcal{H}(T)$ the operator $f(T)$ is defined by the well known analytic calculus (see [9]).

THEOREM 2.7. If $T \in \mathcal{L}(X)$ is totally paranormal, then for each $f \in \mathcal{H}(T)$, Weyl's theorem holds for $f(T)$. 
Proof: We have shown that $T$ has the following properties: $T$ is isoloid (Corollary 2.6), Weyl's theorem holds for $T$ (Theorem 2.5) and ind $(\lambda-T) \leqslant 0$ for all $\lambda \in \Phi(T)$ (Proposition 2.2). Now use Theorem 1 in [15] to derive that Weyl's theorem holds for $f(T)(f \in \mathcal{H}(T))$.

The Weyl spectrum satisfies the one-way spectral mapping theorem ([7, Theorem 2]):

$$
f \in \mathcal{H}(T) \Rightarrow \sigma_{W}(f(T)) \subseteq f\left(\sigma_{W}(T)\right)
$$

This inclusion may be proper $([\mathbf{1}$, Example 3.3$])$. For totally paranormal operators we can say more:

THEOREM 2.8. If $T \in \mathcal{T P \mathcal { N }}(X)$, then

$$
\sigma_{W}(f(T))=f\left(\sigma_{W}(T)\right) \text { for each } f \in \mathcal{H}(T)
$$

Proof: Since ind $(\lambda-T) \leqslant 0$ for each $\lambda \in \Phi(T)$, the assertion follows from [15, Theorem 2].

For the remainder of this section we are concerned with a further important essential spectrum, which we shall introduce now.

An operator $T \in \mathcal{L}(X)$ is called a Kato operator if $T(X)$ is closed and $N(T)$ $\subseteq \bigcap_{n=1}^{\infty} T^{n}(X)$. Let $T \in \mathcal{L}(X)$. In [11] Kato has shown that the set

$$
\rho_{K}(T)=\{\lambda \in \mathcal{C}: \lambda-T \text { is a Kato operator }\}
$$

is open. Since $\rho(T) \subseteq \rho_{K}(T)$ the Kato spectrum

$$
\sigma_{K}(T)=\mathcal{C} \backslash \rho_{K}(T)
$$

is a compact subset of $\sigma(T)$. In [14] we have shown that $\partial \sigma(T) \subseteq \sigma_{K}(T)$ and that $f\left(\sigma_{K}(T)\right)=\sigma_{K}(f(T))$ for all $f \in \mathcal{H}(T)$. For operators in $\mathcal{T P N}(X)$ we have the following result:

THEOREM 2.9. If $T \in \mathcal{L}(X)$ is totally paranormal, then

$$
\rho_{K}(T)=\{\lambda \in \mathcal{C}:(\lambda-T)(X) \quad \text { is closed and } \quad \alpha(\lambda-T)=0\}
$$

Proof: The inclusion $\supseteq$ is clear. Take $\lambda \in \rho_{K}(T)$. Since $T \in \mathcal{T P N}(X)$, we can assume that $\lambda=0$. Put $x \in N(T)$. Since $N(T) \subseteq T(X), x=T y$ for some $y \in X$. Then $T^{2} y=T x=0$, thus

$$
\|x\|^{2}=\|T y\|^{2} \leqslant\left\|T^{2} y\right\|\|y\|=0 .
$$

This shows that $N(T)=\{0\}$. 


\section{LOCAL SPECTRAL PROPERTIES OF TOTALLY PARANORMAL OPERATORS}

Given an operator $T \in \mathcal{L}(X)$, the local resolvent set $\rho_{T}(x)$ of $T$ at the point $x \in X$ is defined as the union of all open subsets $D \subseteq \mathcal{C}$ for which there is a holomorphic function $f: D \rightarrow X$ which satisfies

$$
(\lambda-T) f(\lambda)=x \text { for all } \lambda \in D
$$

Evidently, $\rho_{T}(x)$ is open and $\rho_{T}(x) \supseteq \rho(T)$. The local spectrum $\sigma_{T}(x)$ of $T$ at $x$ is then defined as

$$
\sigma_{T}(x)=\mathcal{C} \backslash \rho_{T}(x)
$$

Clearly, $\sigma_{T}(x)$ is closed and $\sigma_{T}(x) \subseteq \sigma(T)$.

We say that $T \in \mathcal{L}(X)$ has the single-valued extension property, if for every open subset $D \subseteq \mathcal{C}$ the only holomorphic solution $f: D \rightarrow X$ of the equation

$$
(\lambda-T) f(x)=0 \text { for all } \lambda \in D
$$

is the zero function on $D$.

The following proposition is immediate.

Proposition 3.1. Let $T \in \mathcal{L}(X)$ with the single-valued extension property.

(1) If $x \in X$, then there is a unique holomorphic solution $f: \rho_{T}(x) \rightarrow X$ of the equation

$$
(\lambda-T) f(\lambda)=x \quad \text { for all } \quad \lambda \in \rho_{T}(x)
$$

(2) If $x \in X$, then

$$
\sigma_{T}(x)=\emptyset \quad \text { if and only if } x=0 .
$$

Let $T \in \mathcal{L}(X)$ and $F \subseteq \mathcal{C}$. The spectral subspace $X_{T}(F)$ is defined by

$$
X_{T}(F)=\left\{x \in X: \sigma_{T}(x) \subseteq F\right\} .
$$

It is clear that

$$
X_{T}(F)=X_{T}(\sigma(T) \cap F)
$$

and

$$
X_{T}(F) \subseteq X_{T}(G)
$$

whenever $F \subseteq G \subseteq \mathcal{C}$.

Proposition 3.2. Let $T \in \mathcal{L}(X)$ and $\lambda_{0} \in \mathcal{C}$.

(1) $N\left(\lambda_{0}-T\right) \subseteq X_{T}\left(\left\{\lambda_{0}\right\}\right)$.

(2) If $T$ has the single-valued extension property, then

$$
X_{T}\left(\left\{\lambda_{0}\right\}\right)=\left\{x \in X: \lim _{n \rightarrow \infty}\left\|\left(\lambda_{0}-T\right)^{n} x\right\|^{1 / n}=0\right\}
$$


PROOF:

(1) Take $x \in N\left(\lambda_{0}-T\right)$ and define $f: \mathcal{C} \backslash\left\{\lambda_{0}\right\} \rightarrow X$ by $f(\lambda)=\left(\lambda-\lambda_{0}\right)^{-1} x$. Then $(\lambda-T) f(\lambda)=x$ for each $\lambda \neq \lambda_{0}$. Thus $\mathcal{C} \backslash\left\{\lambda_{0}\right\} \subseteq \rho_{T}(x)$, so $\sigma_{T}(x) \subseteq\left\{\lambda_{0}\right\}$.

(2) is shown in [12, Corollary 2.4].

Proposition 3.3. Suppose that $T \in \mathcal{L}(X)$ and that $p(\lambda-T)<\infty$ for each $\lambda \in \mathcal{C}$. Then $T$ has the single-valued extension property.

ProOF: [12, Proposition 1.8].

Proposition 3.4. If $T \in \mathcal{T P N}(X)$, then $T$ has the single-valued extension property.

Proof: Corollary 1.4, Proposition 3.3.

The following result, which is shown in [12, Corollary 4.8], is of central importance for our investigations in the following sections.

Proposition 3.5. If $T \in \mathcal{T P \mathcal { N }}(X)$, then

$$
X_{T}(\{\lambda\})=N(\lambda-T) \text { for all } \lambda \in \mathcal{C}
$$

We close this section with a result, which we need in the final section of this paper.

Proposition 3.6. Let $T \in \mathcal{L}(X), \lambda_{0} \in \mathcal{C}$ and suppose that $\lambda_{0}$ is a simple pole of the resolvent $(\lambda-T)^{-1}$. Then

$$
N\left(\lambda_{0}-T\right)=\left\{x \in X: \lim _{n \rightarrow \infty}\left\|\left(\lambda_{0}-T\right)^{n} x\right\|^{1 / n}=0\right\} .
$$

If in addition $T$ has the single-valued extension property, then

$$
N\left(\lambda_{0}-T\right)=X_{T}\left(\left\{\lambda_{0}\right\}\right)
$$

Proof: [9, Satz 100.2, Satz 101.2] and Proposition 3.2(2).

\section{THE INNER DERIVATION $\delta_{T}$}

In this section we collect some results which we need for the proofs of our results in Section 5.

Given $T \in \mathcal{L}(X)$, the map $\delta_{T}: \mathcal{L}(X) \rightarrow \mathcal{L}(X)$, defined by

$$
\delta_{T}(C)=C T-T C
$$

is called the inner derivation determined by $T$. Evidently, $\delta_{T}$ is a bounded linear operator on $\mathcal{L}(X)$ with $\left\|\delta_{T}\right\| \leqslant 2\|T\|$. 
For the remainder of this paper let $\Psi$ denote the entire function $\Psi: \mathcal{C} \rightarrow \mathcal{C}$ given by

$$
\Psi(z)= \begin{cases}z^{-1}\left(e^{z}-1\right), & \text { if } z \neq 0 \\ 1, & \text { if } z=0\end{cases}
$$

Let $M_{T}=\left\{\lambda \in \sigma\left(\delta_{T}\right): \Psi(\lambda)=0\right\}$.

Proposition 4.1. Let $T \in \mathcal{L}(X)$.

(1) If $C, D \in \mathcal{L}(X), \lambda_{0}, \mu_{0} \in \mathcal{C}, \delta_{T}(C)=\lambda_{0} C$ and $\delta_{T}(D)=\mu_{0} D$, then $\delta_{T}(C D)=\left(\lambda_{0}+\mu_{0}\right) C D$.

(2) If $\lambda \in M_{T}$, the $\lambda$ is a simple zero of $\Psi$ and $\lambda=2 j \pi i$ for some $j \in \mathcal{Z} \backslash\{0\}$.

(3) If $M_{T}=\emptyset$, then $\Psi\left(\delta_{T}\right)$ is an invertible operator.

(4) $M_{T}$ is a finite set and

$$
M_{T} \subseteq\{ \pm 2 \pi i, \pm 4 \pi i, \ldots\}
$$

(5) If $M_{T} \neq \emptyset, M_{T}=\left\{\lambda_{1}, \ldots, \lambda_{p}\right\}$ and $\lambda_{j} \neq \lambda_{k}$ for $j \neq k$, then

$$
N\left(\Psi\left(\delta_{T}\right)\right)=N\left(\delta_{T}-\lambda_{1}\right) \oplus \ldots \oplus N\left(\delta_{T}-\lambda_{p}\right) .
$$

(6) $\sigma\left(\delta_{T}\right)=\{\lambda-\mu: \lambda, \mu \in \sigma(T)\}$.

(7) $e^{\delta_{T}}(C)=e^{-T} C e^{T}$ for all $C \in \mathcal{L}(X)$.

(8) $\Psi\left(\delta_{T}\right)\left(\delta_{T}(C)\right)=e^{-T} C e^{T}-C$ for all $C \in \mathcal{L}(X)$.

ProOF:

(1) Straight forward.

(2) Clear.

(3) Follows from [9, Satz 99.1].

(4) Follows from (2)

(5) Is shown in [17].

(6) Is shown in [9].

(7) Follows from [13, Proposition 6.4.8].

(8) Is a consequence of (7) and the equation $z \Psi(z)=\Psi(z) z=e^{z}-1$.

If $T \in \mathcal{L}(X)$, we define the positive integer $n(T)$ as

$$
n(T)=\min \left\{n \in \mathcal{N}: \frac{r(T)}{\pi}<n\right\}
$$

Proposition 4.2. Let $T \in \mathcal{L}(X)$ and $n=n(T)$. Suppose that $\alpha_{1}, \ldots, \alpha_{n} \in \mathcal{R}$, $C_{1}, \ldots, C_{n} \in \mathcal{L}(X)$ and $\delta_{T}\left(C_{j}\right)=i \alpha_{j} C_{j}(j=1, \ldots, n)$.

(1) If $\alpha_{j} \geqslant 2 \pi$ for $j=1, \ldots, n$, then $C_{1} C_{2} \cdot \ldots \cdot C_{n}=0$. 
(2) If $\alpha_{j} \leqslant-2 \pi$ for $j=1, \ldots, n$, then $C_{1} C_{2} \cdot \ldots \cdot C_{n}=0$.

PRoOF: We only show (1) (the proof for (2) is similar). By Proposition 4.1(1),

$$
\delta_{T}(C)=i\left(\sum_{j=1}^{n} \alpha_{j}\right) C
$$

where $C=C_{1} C_{2} \cdot \ldots \cdot C_{n}$. Assume that $C \neq 0$. Then $i \sum_{j=1}^{n} \alpha_{j} \in \sigma_{p}\left(\delta_{T}\right)$, thus, by Proposition 4.1(6),

$$
i \sum_{j=1}^{n} \alpha_{j}=\lambda-\mu \quad \text { for some } \lambda, \mu \in \sigma(T) \text {. }
$$

This gives

$$
\sum_{j=1}^{n} \alpha_{j}=|\lambda-\mu| \leqslant|\lambda|+|\mu| \leqslant 2 r(T)<2 \pi n,
$$

a contradiction, since $\alpha_{j} \geqslant 2 \pi(j=1, \ldots, n)$.

Proposition 4.3. Let $T \in \mathcal{L}(X), \lambda_{0} \in \mathcal{C}$ and $C \in N\left(\delta_{T}-\lambda_{0}\right)$. Then

$$
C(X) \subseteq X_{T}\left(\sigma\left(T-\lambda_{0}\right) \cap \sigma(T)\right)
$$

Proof: Put $S=T-\lambda_{0}$. For each $\mu \in \rho(S)$ we have

$$
\begin{aligned}
(T-\mu) C(S-\mu)^{-1} & =T C(S-\mu)^{-1}-\mu C(S-\mu)^{-1} \\
& =\left(C T-\lambda_{0} C\right)(S-\mu)^{-1}-\mu C(S-\mu)^{-1} \\
& =C S(S-\mu)^{-1}-\mu C(S-\mu)^{-1} \\
& =C(S-\mu)(S-\mu)^{-1}=C .
\end{aligned}
$$

Therefore, for $\mu \in \rho(S)$ and $x \in X$

$$
(T-\mu) C(S-\mu)^{-1} x=C x .
$$

This shows that for $x \in X$

$$
\rho(S) \subseteq \rho_{T}(C x),
$$

thus $\sigma_{T}(C x) \subseteq \sigma(S)=\sigma\left(T-\lambda_{0}\right)$. Consequently

$$
C x \in X_{T}\left(\sigma\left(T-\lambda_{0}\right)\right)=X_{T}\left(\sigma\left(T-\lambda_{0}\right) \cap \sigma(T)\right) .
$$

Since $x \in X$ was arbitrary, $C(X) \subseteq X_{T}\left(\sigma\left(T-\lambda_{0}\right) \cap \sigma(T)\right)$.

The following proposition is of crucial importance for our investigations in the next section.

Proposition 4.4. Let $T \in \mathcal{T P N}(X)$. Suppose that $\lambda_{0} \in \mathcal{C}, C \in N\left(\delta_{T}-\lambda_{0}\right)$, $\mu_{0} \in \mathcal{C}$ and $\sigma\left(T-\lambda_{0}\right) \cap \sigma(T) \subseteq\left\{\mu_{0}\right\}$. Then

$$
T C=\mu_{0} C
$$


Proof: Use Proposition 4.3 to get

$$
C(X) \subseteq X_{T}\left(\sigma\left(T-\lambda_{0}\right) \cap \sigma(T)\right) \subseteq X_{T}\left(\left\{\mu_{0}\right\}\right) .
$$

Proposition 3.5 shows then that

$$
C x \in X_{T}\left(\left\{\mu_{0}\right\}\right)=N\left(T-\mu_{0}\right) \quad \text { for all } x \in X .
$$

thus $T C x=\mu_{0} C x$ for all $x \in X$.

\section{EXPONENTIALS OF TOTALLY PARANORMAL OPERATORS}

In this section we investigate the operator equations

$$
e^{T}=e^{S} \quad \text { and } \quad e^{T} e^{S}=e^{s} e^{T}
$$

where $T \in \mathcal{T} \mathcal{P N}(X)$ (or $T, S \in \mathcal{T P \mathcal { N }}(X)$ ).

If $T \in \mathcal{L}(X)$, then we say that $\sigma(T)$ is $2 \pi i$-congruence-free if

$$
\sigma(T) \cap \sigma(T+2 k \pi i)=\emptyset \text { for each } k \in \mathcal{Z} \backslash\{0\} .
$$

Hille $[10]$ has shown the following

THEOREM 5.1. Let $T, S \in \mathcal{L}(X)$ and suppose that $\sigma(T)$ is $2 \pi i$-congruence-free. If $e^{T}=e^{S}$, then $T S=S T$.

The next result is due to Wermuth [18] (see also [16] for a very short proof, which uses the derivation $\delta_{T}$ ).

ThEOREM 5.2. Let $T, S \in \mathcal{L}(X)$. Suppose that $\sigma(T)$ and $\sigma(S)$ are $2 \pi i$-congruencefree. If $e^{T} e^{S}=e^{S} e^{T}$, then $T S=S T$.

The object of this section is to obtain results, similar to the above theorems, for totally paranormal operators, where we weaken the property " $2 \pi i$-congruence-free" as follows:

We say that $T \in \mathcal{L}(X)$ has property $(P)$, if

$$
\sigma(T) \cap \sigma(T+2 n \pi i) \subseteq\{n \pi i\} \text { for } n=1,2, \ldots .
$$

REMARKS 5.3. Let $T \in \mathcal{L}(X)$.

(1) It is easy to see that if $T$ has property $(P)$, then

$$
\sigma(T) \cap \sigma(T+2 k \pi i) \subseteq\{k \pi i\} \text { for each } k \in \mathcal{Z} \backslash\{0\} .
$$

(2) If $r(T) \leqslant \pi$, then $T$ has property $(P)$. 
In what follows we shall use the following notations:

$$
\begin{aligned}
i \pi \mathcal{N} & :=\{i \pi, 2 \pi i, 3 \pi i, \ldots\} \\
-i \pi \mathcal{N} & :=\{-i \pi,-2 \pi i,-3 \pi i, \ldots\}, \\
\mathcal{Z}^{*} & =\mathcal{Z} \backslash\{0\}
\end{aligned}
$$

and

$$
i \pi \mathcal{Z}^{*}=(i \pi \mathcal{N}) \cup(-i \pi \mathcal{N})
$$

Proposition 5.4. Let $T \in \mathcal{T} \mathcal{P N}(X)$ have property $(P)$.

(1) If $k \in \mathcal{N}$ and $C \in N\left(\delta_{T}+2 k \pi i\right)$, then

$$
T C=k \pi i C=-C T \text {. }
$$

(2) If $k \in \mathcal{N}$ and $D \in N\left(\delta_{T}-2 k \pi i\right)$, then

$$
T D=-k \pi i D=-D T \text {. }
$$

(3) If $V \in N\left(\Psi\left(\delta_{T}\right)\right)$, then

$$
T V+V T=0
$$

Proof: (1) Put $\lambda_{0}=-2 k \pi i$ and $\mu_{0}=k \pi i$. Then $C \in N\left(\delta_{T}-\lambda_{0}\right)$ and, since $T$ has property $(P)$,

$$
\sigma\left(T-\lambda_{0}\right) \cap \sigma(T)=\sigma(T+2 k \pi i) \cap \sigma(T) \subseteq\left\{\mu_{0}\right\} .
$$

From Proposition 4.4 we derive that $T C=\mu_{0} C=k \pi i C$. Since $C T-T C=-2 k \pi i C$, we get $C T=T C-2 k \pi i C=-k \pi i C^{\prime}=-T C$.

(2) Similar.

(3) By Proposition 4.1(4), (5) there are $\lambda_{1}, \ldots, \lambda_{p} \in\{ \pm 2 \pi i, \pm 4 \pi i, \ldots\}$ and $C_{1}, \ldots, C_{p}$ $\in \mathcal{L}(X)$ such that

$$
V=C_{1}+\ldots+C_{p}
$$

and

$$
C_{j} \in N\left(\delta_{T}-\lambda_{j}\right) \quad(j=1, \ldots, p) .
$$

Use (1) and (2) to see that $T C_{j}+C_{j} T=0(j=1, \ldots, p)$. This shows that $T V+V T=0$. $\square$ Recall that for $T \in \mathcal{L}(X)$ we have denoted by $n(T)$ the smallest positive integer $n$ such that $(r(T)) / \pi<n$.

Proposition 5.5. Let $T \in \mathcal{T P N}(X)$ have property $(P)$ and let $V \in N\left(\Psi\left(\delta_{T}\right)\right)$.

(1) If $(i \pi \mathcal{N}) \cap \sigma_{p}(T)=\emptyset$, then $V^{n(T)}=0$.

(2) If $(-i \pi \mathcal{N}) \cap \sigma_{p}(T)=\emptyset$, then $V^{n(T)}=0$.

(3) If $\left(i \pi \mathcal{Z}^{*}\right) \cap \sigma_{p}(T)=\emptyset$, then $V=0$. 
ProOF: Put $n=n(T)$.

CASE 1. $n=1$. Thus $r(T)<\pi$. Proposition 4.1(6) shows that then $M_{T}=\emptyset$, thus, by Proposition 4.1(5), $\Psi\left(\delta_{T}\right)$ is injective, therefore $V=0$.

CASE 2. $n>1$. Then, use Proposition 4.1,

$$
M_{T} \subseteq\{ \pm 2 \pi i, \pm 4 \pi i, \ldots, \pm 2(n-1) \pi i\}
$$

and

$$
V=U_{1}+\ldots+U_{n-1}+V_{1}+\ldots+V_{n-1}
$$

where $U_{j} \in N\left(\delta_{T}-2 j \pi i\right)$ and $V_{j} \in N\left(\delta_{T}+2 j \pi i\right)(j=1, \ldots, n-1)$. Proposition 5.4 gives

$$
T U_{j}=-j \pi i U_{j} \quad \text { and } T V_{j}=j \pi i V_{j} \quad(j=1, \ldots, n-1) .
$$

(1) If $\left(i \pi \mathcal{N} \cap \sigma_{p}(T)=\emptyset\right.$, then it follows from (*) that $V_{1}=V_{2}=\ldots=V_{n-1}=0$, thus $V=U_{1}+\ldots+U_{n-1}$. The power $V^{n}$ is a sum of products of the form $U_{k_{1}} U_{k_{2}} \cdot \ldots \cdot U_{k_{n}}$, where $U_{k_{\nu}} \in\left\{U_{1}, \ldots, U_{n-1}\right\}$. Therefore

$$
\delta_{T}\left(U_{k_{\nu}}\right)=2 k_{\nu} \pi i U_{k_{\nu}}
$$

and $2 k_{\nu} \pi \geqslant 2 \pi$. From Proposition 4.2 we then conclude that $U_{k_{1}} U_{k_{2}} \cdot \ldots \cdot U_{k_{n}}=0$. Hence $V^{n}=0$.

(2) Similar.

(3) If $\left(i \pi \mathcal{Z}^{*}\right) \cap \sigma_{P}(T)=\Phi,(*)$ shows that $U_{j}=V_{j}=0(j=1, \ldots, n-1)$, thus $V=0$.

Our first result concerning the equation $e^{T} e^{S}=e^{S} e^{T}$ reads as follows:

THEOREM 5.6. Let $T \in \mathcal{T} \mathcal{P N}(X)$ have property $(P)$. Let $S \in \mathcal{L}(X)$ and suppose that $e^{T} e^{S}=e^{S} e^{T}$. Then $T^{2} e^{S}=e^{S} T^{2}$.

Furthermore we have:

(1) If $(i \pi \mathcal{N}) \cap \sigma_{p}(T)=\emptyset$ or $(-i \pi \mathcal{N}) \cap \sigma_{p}(T)=\emptyset$, then

$$
\left(T e^{S}-e^{S} T\right)^{n(T)}=0 .
$$

(2) If $\left(i \pi \mathcal{Z}^{*}\right) \cap \sigma_{p}(T)=\emptyset$, then

$$
T e^{S}=e^{S} T
$$

Proof: By Proposition 4.1(8),

$$
\begin{aligned}
\Psi\left(\delta_{T}\right)\left(\delta_{T}\left(e^{S}\right)\right) & =e^{-T} e^{S} e^{T}-e^{S} \\
& =e^{-T} e^{T} e^{S}-e^{S}=0,
\end{aligned}
$$

thus $V=e^{S} T-T e^{S}=\delta_{T}\left(e^{S}\right) \in N\left(\Psi\left(\delta_{T}\right)\right)$. From Proposition 5.4(3) we derive

$$
\begin{aligned}
0=T V+V T & =T\left(e^{S} T-T e^{S}\right)+\left(e^{S}-T e^{S}\right) T \\
& =e^{S} T^{2}-T^{2} e^{S} .
\end{aligned}
$$


(1) It follows from Proposition 5.5 that $0=V^{n(T)}=\left(e^{S} T-T e^{S}\right)^{n(T)}$.

(2) Use Proposition 5.5(3).

THEOREM 5.7. Let $T, S \in \mathcal{T P N}(X)$ and suppose that $T$ and $S$ have property (P). If $e^{T} e^{S}=e^{S} e^{T}$, then

$$
T^{2} S^{2}=S^{2} T^{2}
$$

Furthermore we have:

(1) If $(i \pi \mathcal{N}) \cap \sigma_{p}(T)=\emptyset$ or $(-i \pi \mathcal{N}) \cap \sigma_{p}(T)=\emptyset$, then $\left(T S^{2}-S^{2} T\right)^{n(T)}=0$.

(2) If $\left(i \pi \mathcal{Z}^{*}\right) \cap \sigma_{p}(T)=\emptyset$ and $\left(i \pi \mathcal{Z}^{*}\right) \cap \sigma_{p}(S)=\emptyset$, then

$$
T S=S T \text {. }
$$

Proof: Theorem 2.1 gives $T^{2} e^{S}=e^{S} T^{2}$. Therefore

$$
\Psi\left(\delta_{S}\right)\left(\delta_{S}\left(T^{2}\right)\right)=e^{-S} T^{2} e^{S}-T^{2}=0
$$

thus $V=T^{2} S-S T^{2}=\delta_{S}\left(T^{2}\right) \in N\left(\Psi\left(\delta_{S}\right)\right)$. Proposition 5.4(3) yields then that

$$
0=S V+V S=S T^{2} S-S^{2} T^{2} S+T^{2} S^{2}-S T^{2} S=T^{2} S^{2}-S^{2} T^{2} .
$$

(1) If we replace $T$ by $S$, we see as above that $U:=\delta_{T}\left(S^{2}\right)=S^{2} T-T S^{2}$ $\in N\left(\Psi\left(\delta_{T}\right)\right.$. Now use Proposition 5.5 to get $U^{n(T)}=0$.

(2) Theorem 5.6(2) shows that $T e^{S}=e^{S} T$, hence

$$
\Psi\left(\delta_{S}\right)\left(\delta_{S}(T)\right)=e^{-S} T e^{S}-T=0 .
$$

Therefore $T S-S T \in N\left(\Psi\left(\delta_{S}\right)\right)$. From Proposition 5.5(3) it follows now that $T S=S T$. $\square$

ThEOREM 5.8. Suppose that $T, S \in \mathcal{L}(X), T+S \in \mathcal{T} \mathcal{P N}(X), T+S$ has property $(P),\left(i \pi \mathcal{Z}^{*}\right) \cap \sigma_{p}(T+S)=\emptyset$ and that

$$
e^{T} e^{S}=e^{T+S}=e^{S} e^{T}
$$

Then $T S=S T$.

Proof: We have

$$
\begin{aligned}
\Psi\left(\delta_{T+S}\right)\left(\delta_{T+S}\left(e^{T}\right)\right) & =e^{-(T+S)} e^{T} e^{T+S}-e^{T} \\
& =e^{-S} e^{-T} e^{T} e^{T+S}-e^{T} \\
& =e^{-S} e^{S} e^{T}-e^{T}=0
\end{aligned}
$$

thus $V=e^{T} S-S e^{T}=e^{T}(T+S)-(T+S) e^{T}=\delta_{T+S}\left(e^{T}\right) \in N\left(\Psi\left(\delta_{T+s}\right)\right)$. By Proposition $5.5(3), N\left(\Psi\left(\delta_{T+S}\right)\right)=\{0\}$, hence $e^{T} S=S e^{T}$. It follows that

$$
\begin{aligned}
\Psi\left(\delta_{T+S}\right)\left(\delta_{T+S}(S)\right) & =e^{-(T+S)} S e^{T+S}-S \\
& =e^{-S} e^{-T} S e^{T} e^{S}-S=0 .
\end{aligned}
$$


Hence $S(T+S)-(T+S) S=S T-T S \in N\left(\Psi\left(\delta_{T+S}\right)\right)=\{0\}$.

Now we are concerned with the equation $e^{T}=e^{S}$.

THEOREM 5.9. Suppose that $T \in \mathcal{T P \mathcal { N }}(X)$ has property $(P), S \in \mathcal{L}(X)$ and that $e^{T}=e^{S}$. Then

$$
T^{2} S=S T^{2}
$$

Furthermore we have:

(1) If $(i \pi N) \cap \sigma_{p}(T)=\emptyset$ or $(-i \pi \mathcal{N}) \cap \sigma_{p}(T)=\emptyset$, then

$$
(T S-S T)^{n(T)}=0 .
$$

(2) If $\left(i \pi \mathcal{Z}^{*}\right) \cap \sigma_{p}(T)=\emptyset$, then

$$
T S=S T \text {. }
$$

ProOF: Since

$$
\Psi\left(\delta_{T}\right)\left(\delta_{T}(S)\right)=e^{-T} S e^{T}-S=e^{-S} S e^{S}-S=0,
$$

$S T-T S \in N\left(\Psi\left(\delta_{T}\right)\right)$. By Proposition 5.4(3) we see that

$$
0=T(S T-T S)+(S T-T S) T=S T^{2}-T^{2} S .
$$

(1) follows from Proposition 5.5(1), (2).

(2) follows from Proposition 5.5(3).

\section{Final Remarks}

In Proposition 3.5 we have seen that if $T \in \mathcal{L}(X)$ is totally paranormal, then

$$
X_{T}(\{\lambda\})=N(T-\lambda) \quad \text { for every } \lambda \in \mathcal{C} .
$$

An inspection of the proofs of Theorem 5.6, 5.7, 5.8 and 5.9 shows that we have only used the property

$$
X_{T}(\{k \pi i\})=N(T-k \pi i) \quad \text { for each } k \in \mathcal{Z}^{*} .
$$

Thus, if we define the class $\mathcal{C}(X)$ by

$$
\mathcal{C}(X)=\left\{T \in \mathcal{L}(X): X_{T}(\{k \pi i\})=N(T-k \pi i) \text { for all } k \in \mathcal{Z}^{*}\right\},
$$

then we have

THEOREM 6.1. Theorems 5.6 through 5.9 remain true if we replace $\mathcal{T P N}(X)$ by $\mathcal{C}(X)$.

EXAMPLE 6.2. Let $T \in \mathcal{L}(X)$ be a scalar-type operator (in the sense of Dunford, see $[4,6])$. Then $T \in \mathcal{C}(X)$. 
This can be seen from [6, Theorem XV.3.4, Theorem XV.8.2] (see also [4, Theorem 5.33, Thereom 11.12]). Furthermore, $T$ has the single-valued extension property [6, Theorem XV.3.2].

An operator $T \in \mathcal{L}(X)$ is said to be meromorphic, if each $\lambda_{0} \in \sigma(T) \backslash\{0\}$ is a pole of the resolvent of $(\lambda-T)^{-1}$. If $T$ is meromorphic, then $\sigma(T)$ has no interior points, thus $T$ has the single-valued extension property.

EXAmpLE 6.3. Let $T \in \mathcal{L}(X)$ be meromorphic. Suppose that $r(T)=\|T\|$,

$$
\sigma(T) \subseteq\{z \in \mathcal{C}:|\operatorname{Im} z| \leqslant \pi\}
$$

and

$$
\sigma(T+2 \pi i) \cap \sigma(T) \subseteq\{i \pi\}
$$

then $T \in \mathcal{C}(X)$ and $T$ has property $(P)$.

Proof: Since (1) and (2) hold, $T$ has property $(P)$. By (1) and Proposition 3.1(2), we only have to show that

$$
X_{T}\left(\left\{\lambda_{0}\right\}\right)=N\left(T-\lambda_{0}\right) \text { for } \lambda_{0} \in\{-i \pi, i \pi\}
$$

since $T$ has the single-value extension property. Take $\lambda_{0} \in\{-i \pi, i \pi\}$. If $\lambda_{0} \in \rho(T)$, then $X_{T}\left(\left\{\lambda_{0}\right\}\right)=N\left(T-\lambda_{0}\right)=\{0\}$. Hence assume that $\lambda_{0} \in \sigma(T)$. From (1) it follows that $\left|\lambda_{0}\right|=r(T)$. Satz in $[9,102.4]$ shows now that $\lambda_{0}$ is a simple pole of $(\lambda-T)^{-1}$, thus by Proposition 3.6

$$
X_{T}\left(\left\{\lambda_{0}\right\}\right)=N\left(T-\lambda_{0}\right)
$$

Corollary 6.4. Suppose that $T \in \mathcal{L}(X)$ is meromorphic and $r(T)=\|T\| \leqslant \pi$. Then $T \in \mathcal{C}(X)$ and $T$ has property $(P)$.

\section{REFERENCES}

[1] S.K. Berberian, 'The Weyl spectrum of an operator', Indiana Univ. Math. J. 20 (1970), 529-544.

[2] L.A. Coburn, 'Weyl's theorem for nonnormal operators', Michigan Math. J. 13 (1966), 285-288.

[3] J.B. Conway, Subnormal operators, Research Notes in Mathematics 51 (Pitman, Boston, Mass., London, 1981).

[4] H.R. Dowson, Spectral theory of linear operators, London Math Society Monographs 12 (Academic Press, London, New York, 1978).

[5] R. Duggirala, 'Paranormal operators', Rev. Colombian Mat. 21 (1987), 135-139.

[6] N. Dunford and J.T. Schwartz, Linear operators, III (J. Wiley and Sons, New York, 1971).

[7] B. Gramsch and D. Lay, 'Spectral mapping theorems for essential spectra', Math. Ann. 192 (1971), 17-32. 
[8] P.R. Halmos, A Hilbert space problem book (Springer-Verlag, Berlin, Heidelberg, New York, 1982).

[9] H. Heuser, Funktionalanalysis, (2nd edition) (B.G. Teubner, Stuttgart, 1986).

[10] E. Hille, 'On roots and logarithms of elements of a complex Banach algebra', Amer. Math. Ann. 136 (1958), 46-57.

[11] T. Kato, 'Perturbation theory for nullity, deficiency and other quantities of linear operators', J. Analyse Math. 6 (1958), 261-322.

[12] K.B. Laursen, 'Operators with finite ascent', Pacific J. Math. 152 (1992), 323-336.

[13] T.W. Palmer, Banach algebras and the general theory of *-algebras. I, Encyclopedia of Maths and its Applications 49 (University of Cambridge, Cambridge, 1994).

[14] Ch. Schmoeger, 'Ein Spektralabbildungssatz.', Arch. Math. (Basel) 55 (1990), 484-489.

[15] Ch. Schmoeger, 'On operators $T$ such that Weyl's theorem hold for $f(T)$ ', Extracta Math. 13 (1998), 27-33.

[16] Ch. Schmoeger, 'Remarks on commuting exponentials in Banach algebras', Proc. Amer. Math. Soc. 127 (1999), 1337-1338.

[17] Ch. Schmoeger, 'Remarks in commuting exponentials in Banach algebras, II', Proc. Amer. Math. Soc. 128 (2000), 3405-3409.

[18] E.M.E. Wermuth, 'A remark on commuting operator exponentials', Proc. Amer. Math. Soc. 125 (1997), 1685-1688.

Mathematisches Institut I

Universität Karlsruhe

D-76128 Karlsruhe

Germany

e-mail: christoph.schmoeger@math.uni-karlsruhe.de 